\title{
Determination of the relationship between core endurance and isokinetic muscle strength of elite athletes
}

\author{
Tuğba Kocahan', Bihter Akınoğlu'** \\ 'The Ministry of Youth and Sports, Sports General Directorship, Department of Health Services, Center of Athlete Training and Health Research, Ankara, Turkey \\ ${ }^{2}$ Department of Physiotherapy and Rehabilitation, Faculty of Health Sciences, Ankara Yıldırım Beyazıt University, Ankara, Turkey
}

Muscle strength and core endurance are both factors contributing to athletes' performance and prevalence of injuries. There are no studies indicating the relationship between muscle strength around the shoulder and knee joints and core endurance.The aim of our study is to determine the relationship between core endurance and isokinetic muscle strength of knees and shoulders of elite athletes. Seventy-one elite athletes (weight lifting, boxing, taekwondo, biathlon, and ice skating) (age, $18.13 \pm 2.9$ years) were included in the study. Isokinetic muscle strength of shoulder internal-external rotation and knee flexion-extension were determined by using an Isomed 2000 device. Core endurance of athletes was assessed using the Mcgill Core Endurance Tests. There was a relationship between the shoulder internal rotation and external rotation peak torque/body weight (PT/W) and all endurance tests except extension endurance tests. There was also a relationship between knee flexion PT/W and all core endurance tests. While there was a relationship between knee extension PT/W and extension endurance and the lateral bridge test, this relationship was not found with the flexor endurance test. These results indicate that the upper and lower extremity muscle strength and core endurance of athletes are related with each other and must be evaluated and trained as a whole with each other.

Keywords: Isokinetic muscle strength, Upper extremity, Lower extremity, Core endurance, Athletes

\section{INTRODUCTION}

Muscle strength contributes to performing many activities unique to sports including jumping and direction change and affect athletic performance. Furthermore, the core muscles are one of the most important components of these muscle activations (Suchomel et al., 2016).

The core muscles consists of the diaphragm at the upper section, abdominal and oblique muscles at the front and lateral sections, paraspinal and gluteal muscles at the back, and pelvic floor and hip junction at the lower section (Akuthota and Nadler, 2004). This muscular structure distributes the forces that act and allows effective body movements; hence it provides strength and movement formation in the extremities (Huxel Bliven and Anderson, 2013). Moreover, it generates a stabilization effect on the body and spine similar to corset (Rivera, 2016).

Multiple functions of the core mechanism have directed researchers to investigate the relationship between this muscular structure and the surrounding structures. These studies showed that the scapula, shoulder muscles and lower extremity muscles must work coherently with the core muscles for controlled movements (Hodges and Richardson, 1997a; Hodges and Richardson, 1997b). There are numerous studies conducted on the role of core stability to prevent especially back pains of athletes and injuries of lower extremities (Abdelraouf and Abdel-Aziem, 2016; Hodges et al., 1997; Hölmich et al., 2010; Hübscher et al., 2010; Hungerford et al., 2003; Rickman et al., 2012). It has been shown in the literature that lower extremity injuries influence core stability measurements. Moreover, the insufficiency in core stability augments lower extremity injury risks (Willson et al., 2005). Much research in-
${ }^{*}$ Corresponding author: Bihter Akınoğlu (iD https://orcid.org/0000-0002-8214-7895 Department of Physiotherapy and Rehabilitation, Faculty of Health Sciences, Ankara Yildırım Beyazıt University, Ankara, Turkey

Tel: +90-312-305-1555, Fax: +90-312-596-7048, E-mail: rgkardelen@yahoo.com Received: March 13, 2018 / Accepted: April 28, 2018
This is an Open Access article distributed under the terms of the Creative Commons Attribution Non-Commercial License (http://creativecommons.org/licenses/by-nc/4.0/) which permits unrestricted non-commercial use, distribution, and reproduction in any medium, provided the original work is properly cited. 
vestigated the relationship between core stability and athletic performance in various branches including dancers, joggers, etc. (Abt et al., 2007; Lust et al., 2009; Nesser et al., 2008; Saeterbakken et al., 2011). Furthermore, studies investigating the relationship between upper extremities and core muscles have been reported in the literature recently (Saeterbakken et al., 2011; Radwan et al., 2014). Radwan et al. (2014) studied the relationship between core instability and shoulder dysfunction and found that lack of balance and stability increased shoulder dysfunction.

Although the relation between the hip muscle and core stabilization is identified, there is no study in the literature reporting the relationship between core endurance and isokinetic muscle strength of muscles surrounding the knee and shoulder. As a result, we planned our study to investigate whether there is a relationship between the endurance of core muscles and isokinetic strength of shoulder internal-external rotator muscles and knee flexor-extensor muscles. Revealing the mentioned relationship will lead to determination of appropriate exercise and training programs and prevention of athlete injuries in the long term.

\section{MATERIALS AND METHODS}

The aim of our study is to determine the relationship between core endurance and knee and shoulder isokinetic muscle strength of elite athletes.

\section{Ethical clerance}

The study was approved and supported by the Social and Human Ethics Committee of Ankara Yıldırım Beyazıt University (11/01:272).

\section{Setting}

The Ministry of Youth and Sports, Sports General Directorship, Department of Health Services, Center of Athlete Training and Health Research, Ankara, Turkey.

\section{Participants}

Seventy-one professional national elite athletes in various sports branches (box [ $\mathrm{n}=15]$, weight lifting [ $\mathrm{n}=18]$, biathlon [ $\mathrm{n}=17]$, ice skating [ $\mathrm{n}=13$ ], taekwondo $[\mathrm{n}=8]$ ) who gave informed concent to participation for an assessment and did not have any orthopedic or sport injuries participated in our study. The athletes were informed about the study purpose and process Volunteerism was the basis in the study and consents of the participants were received

\section{Procedure}

Descriptive information of the athletes including their age, height, body weight and sports type, etc. were recorded. Isokinetic muscle strength was assessed by using the ISOMED 2000 (D. \& R. Ferstl GmbH, Hemau, Germany) device. The athletes were asked to do jogging for 10 minutes prior to the assessment. The height and body weight of the athletes were entered in the device and personal adjustments were made. The joint movement-spaces were determined as the user manual of the device and the device was setup based on the angles determined by a pretest at a very low speed. Test angles for shoulder internal rotation (IR)/external rotation (ER) movements were determined as $0^{\circ} \mathrm{ER}$ and $90^{\circ} \mathrm{IR}$ at scapular neutral position (the definitions accepted in the Isomed 2000 device and by the used protocols). Test angles were determined as $90^{\circ}$ flexion and $0^{\circ}$ extension for knee flexion/extension movements. Both tests were assessed in the sitting position, the gravity effect was zeroed by using the device and by giving visual and verbal feedback. In the assessment protocol, the athletes were asked to repeat the movement 3 times as submaximal for warming up and understanding the movement. Following the warmup movements, they relaxed for $1 \mathrm{~min}$, after relaxation they repeated IR/ER movement five times at $60^{\circ} / \mathrm{sec}$ of speed and the test was completed for one side (Shaw et al., 2009).

The assessments were carried out bilaterally for each joint and applied first for the dominant side and after the athletes getting relax, it was applied for the nondominant side. After 1 day with a day interval, the same protocol was applied for flexion-extension movements in the knee joint. All measurements were performed by the same researcher with 3 years of experience during a single test session. And all the procedure was done the same for all the athletes. Muscle strength was evaluated in terms of peak torque and peak torque/body weight (PT/W percent of torque produced per $\mathrm{kg}$ of body weight) estimated with five repeated-measurements at an angular speed of $60^{\circ} / \mathrm{sec}$ (Shaw et al., 2009).

Core endurance was assessed by using the Mcgill Core Endurance Tests. The anterior core endurance was assessed by using the flexor endurance test. The test was initiated with the participant's hands crossed at the shoulder; the knee and hip joints at $90^{\circ}$ flexion; the feet stabilized by the research assistant physiotherapist. The participant's body was flexed until the lower end of the scapula rose from the bed and the duration of maintaining this position was recorded by using a stop watch (Fig. 1). The extensor endurance test was used to test the erector spina and multifidus. During this test, the participant's body was hanging down from the table as the spina iliaca anterior superiors aligned with the table 


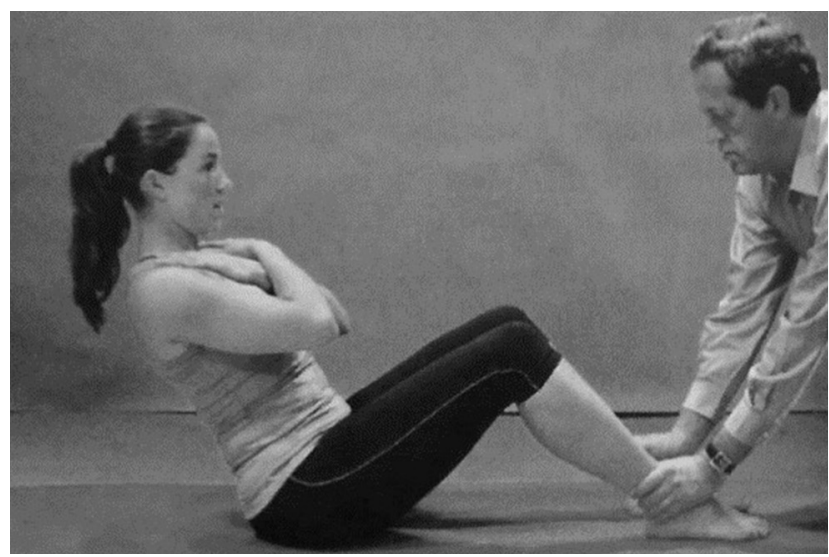

Fig. 1. Flexor endurance test. Reprinted from Clinical Sports Medicine (p.214), by P. Brukner and K. Khan, 2012, Australia: McGraw-Hill Education Pty Ltd. Copyright 2012 by McGraw-Hill Education Pty Ltd. Reprinted with permission.

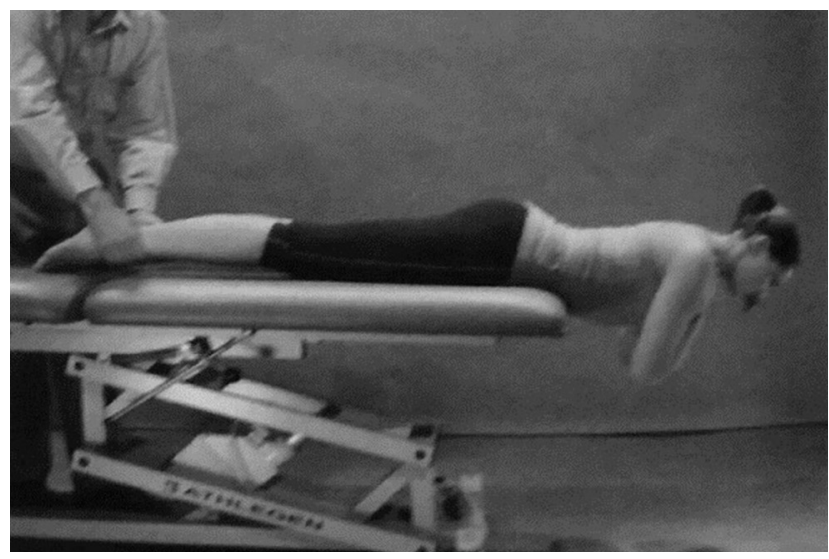

Fig. 2. Static trunk endurance test. Reprinted from Clinical Sports Medicine (p.214), by P. Brukner and K. Khan, 2012, Australia: McGraw-Hill Education Pty Ltd. Copyright 2012 by McGraw-Hill Education Pty Ltd. Reprinted with permission.

edge, the hands were crossed at the shoulders and the feet were in the supported position. The test was initiated when horizontality was achieved and the duration to maintain this position was recorded by using a stop watch (Fig. 2). The lateral bridge test was performed to test lateral core muscles. This test was performed in a side-lying position with the legs extended with one foot in front of the other foot and the body at in a straight line by lifting on the front arm of the lying side. The duration to maintain this position was recorded by using a stop watch. This test was assessed for the dominant side first and then for the nondominant side (Fig. 3) All the Mcgill Core Endurance Tests was explained to athletes first than the tests performed once (Cowan, 2012).

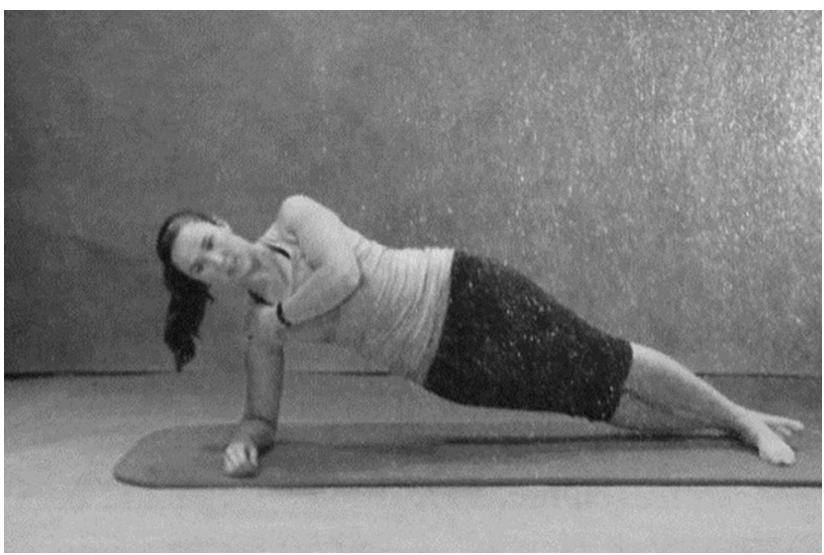

Fig. 3. Lateral bridge test. Reprinted from Clinical Sports Medicine (p.214), by P. Brukner and K. Khan, 2012, Australia: McGraw-Hill Education Pty Ltd. Copyright 2012 by McGraw-Hill Education Pty Ltd. Reprinted with permission.

Table 1. Descriptive features of athletes

\begin{tabular}{lc}
\hline Variable & Mean \pm SD \\
\hline Age $(\mathrm{yr})$ & $18.13 \pm 2.9$ \\
Height $(\mathrm{cm})$ & $172 \pm 0.08$ \\
Weight $(\mathrm{kg})$ & $68.21 \pm 15.0$ \\
Body mass index $\left(\mathrm{kg} / \mathrm{m}^{2}\right)$ & $22.77 \pm 3.5$ \\
Sport year & $9.2 \pm 2.7$ \\
\hline
\end{tabular}

$\mathrm{SD}$, standard deviation.

Table 2. Descriptive features of isokinetic muscle strength and core endurance

\begin{tabular}{|c|c|c|}
\hline Variable & Dominant & Nondominant \\
\hline \multicolumn{3}{|l|}{ Muscle strength $\left(60^{\circ} / \mathrm{sec}\right)$} \\
\hline \multicolumn{3}{|l|}{ Knee } \\
\hline Flexion PT (N/m) & $113.76 \pm 30.58$ & $109.32 \pm 29.40$ \\
\hline Flexion PT/W (N/m/kg) & $1.65 \pm 0.37$ & $1.59 \pm 0.38$ \\
\hline Extension PT (N/m) & $214.48 \pm 54.96$ & $202.03 \pm 59.11$ \\
\hline Extension PT/W (N/m/kg) & $3.06 \pm 0.84$ & $2.91 \pm 0.89$ \\
\hline \multicolumn{3}{|l|}{ Shoulder } \\
\hline Internal rotation PT (N/m) & $59.01 \pm 20.20$ & $56.40 \pm 17.86$ \\
\hline Internal rotation PT/W (N/m/kg) & $0.87 \pm 0.27$ & $0.81 \pm 0.25$ \\
\hline External rotation PT (N/m) & $33.86 \pm 10.12$ & $30.61 \pm 9.80$ \\
\hline External rotation PT/W (N/m/kg) & $0.53 \pm 0.35$ & $0.48 \pm 0.35$ \\
\hline \multicolumn{3}{|l|}{ Core endurance tests (sec) } \\
\hline Lateral flexion test & $101.90 \pm 47.99$ & $97.64 \pm 49.94$ \\
\hline Flexor endurance test & \multicolumn{2}{|c|}{$173.56 \pm 162.45$} \\
\hline Extensor endurance test & \multicolumn{2}{|c|}{$144.69 \pm 71.78$} \\
\hline
\end{tabular}

Values are presented as mean \pm standard deviation.

PT, peak torque; PT/W, peak torque/body weight.

\section{Statistical analysis}

Data were analyzed by using IBM SPSS Statistics ver. 22.0 (IBM 
Table 3. The correlation between the upper extremity isokinetic muscle strength and core endurance

\begin{tabular}{|c|c|c|c|c|c|c|c|c|}
\hline \multirow{2}{*}{ Variable } & \multicolumn{2}{|c|}{ Flexor endurance test } & \multicolumn{2}{|c|}{ Extensor endurance test } & \multicolumn{2}{|c|}{ Lateral bridge test (DM) } & \multicolumn{2}{|c|}{ Lateral bridge test (NDM } \\
\hline & $r$ & $P$ & $r$ & $P$ & $r$ & $P$ & $r$ & $P$ \\
\hline DM-IR-60\% /sec PT & 0.073 & 0.545 & -0.146 & 0.224 & 0.097 & 0.423 & -0.144 & 0.231 \\
\hline NDM-IR-60\% $/ \mathrm{sec}$ PT & 0.021 & 0.864 & -0.168 & 0.160 & -0.176 & 0.142 & -0.157 & 0.192 \\
\hline DM-ER-60\% $/$ sec PT & 0.087 & 0.473 & -0.089 & 0.458 & -0.097 & 0.419 & -0.159 & 0.186 \\
\hline NDM-ER-60\% $/$ sec PT & 0.044 & 0.714 & -0.089 & 0.463 & -0.164 & 0.172 & -0.211 & 0.078 \\
\hline DM-IR-60\% / sec PT/W & $0.354^{*}$ & 0.002 & 0.122 & 0.310 & $0.242^{*}$ & 0.042 & $0.301^{*}$ & 0.011 \\
\hline NDM-IR-60\%/sec PT/W & $0.267^{*}$ & 0.024 & 0.094 & 0.436 & $0.220^{*}$ & 0.046 & $0.308^{*}$ & 0.009 \\
\hline DM-ER-60\%/sec PT/W & $0.418^{*}$ & 0.000 & 0.210 & 0.079 & $0.352^{*}$ & 0.003 & $0.375^{*}$ & 0.001 \\
\hline NDM-ER-60\%/sec PT/W & $0.321^{*}$ & 0.006 & 0.158 & 0.188 & $0.299^{*}$ & 0.011 & $0.238^{*}$ & 0.046 \\
\hline
\end{tabular}

$\mathrm{DM}$, dominant side; ND, nondominant side; IR, internal rotation; ER, external rotation; PT, peak torque; PT/W, peak torque/ body weight. ${ }^{*} P<0.05$.

Table 4. The correlation between isokinetic muscle strength of the lower extremity and core endurance

\begin{tabular}{|c|c|c|c|c|c|c|c|c|}
\hline \multirow{2}{*}{ Variable } & \multicolumn{2}{|c|}{ Flexor endurance test } & \multicolumn{2}{|c|}{ Extensor endurance test } & \multicolumn{2}{|c|}{ Lateral bridge test (DM) } & \multicolumn{2}{|c|}{ Lateral bridge test (NDM } \\
\hline & $r$ & $P$ & $r$ & $P$ & $r$ & $P$ & $r$ & $P$ \\
\hline DM-KF-60\% $/ \mathrm{sec}$ PT & 0.167 & 0.165 & 0.052 & 0.669 & -0.071 & 0.557 & -0.118 & 0.329 \\
\hline NDM- KF-60\% $/ \mathrm{sec}$ PT & 0.189 & 0.115 & 0.045 & 0.712 & 0.030 & 0.806 & -0.069 & 0.569 \\
\hline DM-KE-60\% $/ \mathrm{sec}$ PT & 0.068 & 0.572 & 0.003 & 0.977 & -0.009 & 0.940 & -0.139 & 0.247 \\
\hline NDM-KE-60\% /sec PT & 0.122 & 0.310 & 0.057 & 0.634 & -0.016 & 0.893 & -0.054 & 0.655 \\
\hline DM-KF-60\%/sec PT/W & $0.286^{*}$ & 0.016 & $0.300^{*}$ & 0.011 & $0.257^{*}$ & 0.031 & $0.250^{*}$ & 0.035 \\
\hline NDM- KF-60 \%sec PT/W & $0.293^{*}$ & 0.013 & $0.257^{*}$ & 0.031 & $0.295^{*}$ & 0.013 & $0.251^{*}$ & 0.035 \\
\hline DM-KE-60 \% sec PT/W & 0.214 & 0.073 & $0.247^{*}$ & 0.049 & $0.318^{*}$ & 0.007 & $0.243^{*}$ & 0.041 \\
\hline NDM-KE-60\%/sec PT/W & 0.225 & 0.059 & $0.265^{*}$ & 0.026 & $0.286^{*}$ & 0.016 & $0.270^{*}$ & 0.023 \\
\hline
\end{tabular}

$\mathrm{DM}$, dominant side; ND, nondominant side; PT, peak torque; PT/W, peak torque/ body weight; $K F$, knee flexion; KE, knee extension. ${ }^{*} P<0.05$.

Co., Armonk, NY, USA) and the descriptive statistics of all variables were estimated. The results were reported as mean \pm standard deviation. The Shapiro-Wilk test was used according to the sample number to determine whether the data was normally distributed. Spearman correlation analysis was used to determine the relationship between core endurance and isokinetic muscle strength of the participants. The statistically significance value was accepted as $P<0.05$ for data analysis.

\section{RESULTS}

The descriptive features of participants are shown in Table 1 . The descriptive features of participants' isokinetic muscle strength and core endurance are shown in Table 2. The correlation between the upper extremity isokinetic muscle strength and core endurance are shown in Table 3. While there was a relation between the shoulder IR-ER PT/W and flexor endurance and lateral bridge tests $(P<0.05)$ there was not any relation between the shoulder
IR-ER PT and core endurance test $(P>0.05)$ (Table 3).

The correlation between isokinetic muscle strength of the lower extremity and core endurance are shown in Table 4. It was found that, there was a relation between knee flexion PT/W and all core endurance tests $(P<0.05)$. Also there there was also a relationship between knee extension PT/W and the extensor endurance test and lateral bridge endurance tests $(P<0.05)$, there was no relationship between knee flexion-extension PT and the core endurance test $(P<0.05)$ (Table 4$)$.

\section{DISCUSSION}

The effects of muscle strength on athletic performance and the role of core muscles to reduce sports injuries was considered to conduct our research. The aim was to determine the relationship between upper and lower extremity muscle strength and core endurances of athletes and to provide insight into training programs of athletes based on this information and investigated the rela- 
tionship between shoulder IR-ER and knee flexor-extensor isokinetic muscle strength and core endurance tests.

The results of our study indicated that there is no relationship between core endurances and PT values which is one of the important results of isokinetic muscle strength measurement. In the majority of studies conducted by isokinetic measurement methods in the literature, PT values are taken into consideration. But it is stated that the PT/W value is also a significant parameter. As a result of our study, this may be the reason for the lack of significant relationships between PT value and core endurances.

The core muscles, which function as a corset on the body and spine, contract before a sudden shoulder movement thus, strength and movement is achieved in the extremities (Huxel Bliven and Anderson, 2013). In our study, we found that there was a relationship between shoulder IR-ER PT/W and flexor endurance test and lateral bridge endurance tests. However, there was no relationship between the extensor endurance test and IR-ER PT/W. There were no studies found in the literature investigating the relationship between the shoulder joint isokinetic muscle strength and core muscles endurance. But this relation can be explained by Hodges and Richardson's study. In this study the researchers found that abdominal contraction occurs prior to sudden shoulder movements in order to control the expected effect of the reactive strength that is generated by movement on postural balance and spinal stability (Hodges and Richardson, 1997b). Similar to this study, the results of our study support the relationship between core muscles and upper extremity muscle strength because of the physiological foundations and anatomical locations of core muscles.

There are studies reported in the literature investigating the relationship between the lower extremity muscle strength and endurance of core muscles. One of these studies was conducted by Gordon et al. (2013) on 45 healthy Lakros players. In this study, the relationship between hip ER strength and balance and core muscle strength was investigated. Hip ER isometric muscle strength was measured by a manual dynamometer and core muscle strength was evaluated by a stabilizer. The results of the study indicated that there was no significant relationship between hip ER isometric muscle strength and core muscle strength (Gordon et al., 2013). Moreover, in a study conducted by Ambegaonkar et al. (2014) on 40 healthy athletes, the relationship between the endurance of core muscles and isometric muscle strength of hip muscles was investigated. Isometric muscle strength of hip abductor, ER, flexor and extensor muscles was evaluated by a manual dynamometer and the endurance of core muscles was evaluated by using the extensor endurance test, flexor endurance test and lateral bridge test.
As a result of the mentioned study, it was determined that there was a significant relationship between core extensor endurance and bilateral hip ER isometric muscle strength. In our study, the relationship between knee flexion-extension isokinetic muscle strength and endurance of core muscles was investigated because quadriceps and hamstring muscles play a major role in movement of lower extremities and also contribute to pelvis stabilization since they adhere to the pelvis and its surrounding region similar to core muscles. Also, there is a relationship between the core endurance and prevalence of sport injuries and the most injured regions of the athletes are the knee and the shoulder (Green et al., 2007). Therefore, we wanted to explain this relation to identify the factors that are influence injury. The results of our studies indicated that there was a relationship between PT/W of knee flexors and all core endurance test results and also knee extensors PT/ W were related with all core endurance tests except the flexor endurance test. This result is similar to the relationship between lower extremity muscle strength and core muscle endurance reported in the literature (Cinar-Medeni et al., 2015). This showed that muscle strength of knee flexors, which play a crucial role to achieve postural stability was more related with the endurance of core muscles.

The limitation in our study was that muscles were not evaluated in isolation by the measurement of the endurance of core muscles and isokinetic tests since other muscle groups may have contracted actively. Isokinetic tests must be performed in combination with electromyography and at various angular speeds, and studies are needed to investigate the relationship between core muscle endurance and muscle strength of lower and upper extremities in specific sports branches.

In conclusion, our result indicates that the upper and lower extremity muscle strength and core endurance of athletes are related with each other and must be evaluated and trained as a whole. In the literature the relationship between lower extremity muscle strength, especially the hip muscle and core endurance have been proven and the physiologic mechanism of this relationship is disclosed. In a similar way, the relationship between the strength of muscle of surrounding the knee and the shoulder determined with our study.

\section{CONFLICT OF INTEREST}

No potential conflict of interest relevant to this article was reported. 


\section{REFERENCES}

Abdelraouf OR, Abdel-Aziem AA. The relationship between core endurance and back dysfunction in collegiate male athletes with and without nonspecific low back pain. Int J Sports Phys Ther 2016;11:337-344.

Abt JP, Smoliga JM, Brick MJ, Jolly JT, Lephart SM, Fu FH. Relationship between cycling mechanics and core stability. J Strength Cond Res 2007;21:1300-1304.

Akuthota V, Nadler SF. Core strengthening. Arch Phys Med Rehabil 2004; 85(3 Suppl 1):S86-92.

Ambegaonkar JP, Mettinger LM, Caswell SV, Burtt A, Cortes N. Relationships between core endurance, hip strength, and balance in collegiate female athletes. Int J Sports Phys Ther 2014;9:604-616.

Cinar-Medeni O, Baltaci G, Bayramlar K, Yanmis I. Core stability, knee muscle strength, and anterior translation are correlated with postural stability in anterior cruciate ligament-reconstructed patients. Am J Phys Med Rehabil 2015;94:280-287.

Cowan A. Core stability. In: Brukner P, Khan K, editors, Clinical sports medicine. 4th ed. Sydney (Australia): McGraw-Hill; 2012. p. 210-226.

Gordon AT, Ambegaonkar JP, Caswell SV. Relationships between core strength, hip external rotator muscle strength, and star excursion balance test performance in female lacrosse players. Int J Sports Phys Ther 2013;8:97-104.

Green CM, Petrou MJ, Fogarty-Hover ML, Rolf CG. Injuries among judokas during competition. Scand J Med Sci Sports 2007;17:205-210.

Hodges PW, Gandevia SC, Richardson CA. Contractions of specific abdominal muscles in postural tasks are affected by respiratory maneuvers. J Appl Physiol (1985) 1997;83:753-760.

Hodges PW, Richardson CA. Contraction of the abdominal muscles associated with movement of the lower limb. Phys Ther 1997a;77:132-142.

Hodges PW, Richardson CA. Relationship between limb movement speed and associated contraction of the trunk muscles. Ergonomics 1997b;40: 1220-1230.

Hölmich P, Larsen K, Krogsgaard K, Gluud C. Exercise program for pre- vention of groin pain in football players: a cluster-randomized trial. Scand J Med Sci Sports 2010;20:814-821.

Hübscher M, Zech A, Pfeifer K, Hänsel F, Vogt L, Banzer W. Neuromuscular training for sports injury prevention: a systematic review. Med Sci Sports Exerc 2010;42:413-421.

Hungerford B, Gilleard W, Hodges P. Evidence of altered lumbopelvic muscle recruitment in the presence of sacroiliac joint pain. Spine (Phila Pa 1976) 2003;28:1593-1600.

Huxel Bliven KC, Anderson BE. Core stability training for injury prevention. Sports Health 2013;5:514-522.

Lust KR, Sandrey MA, Bulger SM, Wilder N. The effects of 6-week training programs on throwing accuracy, proprioception, and core endurance in baseball. J Sport Rehabil 2009;18:407-426.

Nesser TW, Huxel KC, Tincher JL, Okada T. The relationship between core stability and performance in division I football players. J Strength Cond Res 2008;22:1750-1754.

Radwan A, Francis J, Green A, Kahl E, Maciurzynski D, Quartulli A, Schultheiss J, Strang R, Weiss B. Is there a relation between shoulder dysfunction and core instability? Int J Sports Phys Ther 2014;9:8-13.

Rickman AM, Ambegaonkar JP, Cortes N. Core stability: implications for dance injuries. Med Probl Perform Art 2012;27:159-164.

Rivera CE. Core and Lumbopelvic Stabilization in Runners. Phys Med Rehabil Clin N Am 2016;27:319-337.

Saeterbakken AH, van den Tillaar R, Seiler S. Effect of core stability training on throwing velocity in female handball players. J Strength Cond Res 2011;25:712-718.

Shaw I, Shaw BS, Cilliers JF, Goon DT. Influence of visual feedback on knee extensor isokinetic concentric and eccentric peak torque. Afr J Phys Health Educ Recreat Dance 2009;15:257-264.

Suchomel TJ, Nimphius S, Stone MH. The Importance of Muscular Strength in Athletic Performance. Sports Med 2016;46:1419-1449.

Willson JD, Dougherty CP, Ireland ML, Davis IM. Core stability and its relationship to lower extremity function and injury. J Am Acad Orthop Surg 2005;13:316-325. 